\title{
Response inhibition is linked to emotional devaluation: behavioural and electrophysiological evidence
}

\author{
Monika Kiss ${ }^{1, *}$, Jane E. Raymond ${ }^{2}$, Nikki Westoby ${ }^{2}$, Anna C. Nobre ${ }^{3}$ and Martin Eimer ${ }^{1}$ \\ 1. School of Psychology, Birkbeck College, University of London, London, UK \\ 2. University of Wales, Bangor, UK \\ 3. University of Oxford, Oxford, UK \\ Edited by: $\quad$ Francisco Barcelo, University of Illes Balears, Spain \\ Reviewed by: Antoni Rodriguez-Fornells, University of Barcelona, Spain \\ Luiz Pessoa, Indiana University, USA
}

\begin{abstract}
To study links between the inhibition of motor responses and emotional evaluation, we combined electrophysiological measures of prefrontal response inhibition with behavioural measures of affective evaluation. Participants first performed a Go-Nogo task in response to Asian and Caucasian faces (with race determining their Go or Nogo status), followed by a trustworthiness rating for each face. Faces previously seen as Nogo stimuli were rated as less trustworthy than previous Go stimuli. To study links between the efficiency of response inhibition in the Go-Nogo task and subsequent emotional evaluations, the Nogo N2 component was quantified separately for faces that were later judged to be high versus low in trustworthiness. Nogo N2 amplitudes were larger in response to low-rated as compared to high-rated faces, demonstrating that trial-by-trial variations in the efficiency of response inhibition triggered by Nogo faces, as measured by the Nogo N2 component, co-vary with their subsequent affective evaluation. These results suggest close links between inhibitory processes in top-down motor control and emotional responses.
\end{abstract}

Keywords: emotion, response inhibition, event-related brain potentials, Nogo N2 component, cognitive control

\section{INTRODUCTION}

When confronted with a complex sensory environment where multiple objects and events are simultaneously present, organisms have to rely on mechanisms that enable them to select task-relevant information, and to ignore information that is not relevant to their current goals. While this type of selection has traditionally been thought to be mediated by processes of selective attention, it has now become clear that emotional processes also play an important role in the prioritization of sensory information for perception, cognition, and motor control. The presence of emotionally significant stimuli can modulate sensory processing (e.g. Lang et al., 1990, 1998), memory and decision making (see Cacioppo and Gardner, 1999, for a review), as well as attentional processes (e.g. Eastwood et al., 2001; Fox et al., 2001; Lang et al., 1997). Functional brain imaging studies have revealed strong links between brain mechanisms involved in attention and emotion (e.g. Bush et al., 2000; Pessoa and Ungerleider, 2004; Vuilleumier et al., 2001), which further underlines their joint role in the selective control of information processing.

*Correspondence: Monika Kiss, School of Psychology, Birkbeck College, University of London, Malet Street, London WC1E 7HX, UK. e-mail: m.kiss@bbk.ac.uk

Received: 16 May 2008; paper pending published: 28 June 2008; accepted: 19 September 2008; published online: 03 October 2008.

Citation: Front. Hum. Neurosci. (2008) 2: 13. doi: 10.3389/neuro.09.013.2008

Copyright $\odot 2008$ Kiss, Raymond, Westoby, Nobre and Eimer. This is an open-access article subject to an exclusive license agreement between the authors and the Frontiers Research Foundation, which permits unrestricted use, distribution, and reproduction in any medium, provided the original authors and source are credited.
Links between emotion and attention have been demonstrated in numerous studies showing that emotionally salient visual events can bias selective attention (e.g. Esteves et al., 1994; Mogg and Bradley, 1999; Öhman et al., 2001). However, the question of whether such links might also operate in reverse (i.e. attention affecting emotional responses) has only recently been addressed. Raymond et al. (2003) first demonstrated systematic effects of attentional selection of stimuli on their subsequent emotional evaluation. In their study, two coloured abstract images were shown in the left and right visual field, and participants reported the location of one of these (previously designated as the target), while ignoring the other. Subsequently, they had to evaluate one of these images, or a previously unseen novel image, on a 'cheerfulness' scale. Ratings for previously ignored distractors were more negative than ratings for previous targets or novel images but ratings for targets were not different from ratings for novel images. In other words, stimuli were devalued as a consequence of being ignored.

Raymond et al. (2003) explained these findings by proposing that attentional inhibition was applied to task-irrelevant distractors during the initial target selection task and that a code for this inhibitory state was then associated with the distracting item. Later during evaluation, inhibition was reinstated, resulting in negative affective judgements for distractors. A key component of this theoretical view is that the inhibitory state invoked for the purpose of selection becomes associated in memory with the to-be-ignored feature (Kessler and Tipper, 2004; Tipper et al., 2003) Subsequent experiments supporting this inhibition-based view showed that post-attentional devaluation 
only occurred for distractor stimuli that were spatially proximal to targets (Raymond et al., 2005), i.e. most likely to attract attentional inhibition (Chen et al., 2008), and that had features that directly interfered with the prior target selection task (Goolsby et al., 2008). An alternative explanation concerns the notion of perceptual fluency (Griffiths and Mitchell, 2008). This idea proposes that changes in evaluative responses to stimuli are brought about by prior exposure that passively produces changes in the ease or fluency of perceptual processing which is then interpreted by affective mechanisms (Reber et al., 1998). However, this idea seems implausible to explain the devaluation (as opposed to enhanced evaluation) of stimuli (especially relative to novel stimuli). Moreover, prolonged exposure to stimuli reduces (rather than enhances) their subsequent evaluations if those stimuli are known to be distractors in a visual search task (Fenske et al., 2005).

In a recent event-related brain potential (ERP) study (Kiss et al., 2007), we obtained independent electrophysiological evidence that the efficiency of attentional distractor suppression is directly linked to their subsequent devaluation. On each trial, pairs of faces were presented, and participants had to select one of these on the basis of gender (with male or female faces designated as targets in different blocks). Subsequently, they had to rate either the target or distractor face in terms of its trustworthiness. As expected, distractors were rated as less trustworthy than targets (see also Goolsby et al., 2008). Critically, we measured the $\mathrm{N} 2 \mathrm{pc}$ component during the initial attentional selection stage. The N2pc is elicited at posterior electrodes contralateral to the side of task-relevant visual events at about $200 \mathrm{~ms}$ after stimulus onset, and reflects the selective attentional processing of targets versus distractors (Eimer, 1996; Luck and Hillyard, 1994; Woodman and Luck, 1999). Large N2pc amplitudes indicate fully focused attention and effective distractor inhibition, while smaller and delayed N2pc components are linked to a more diffuse attentional state. To demonstrate direct links between the efficiency of selective attention and subsequent emotional responses, we computed the N2pc component on each trial as a function of subsequent evaluative judgements, and found that the affective ratings of distractor faces, but not of target faces, covaried with attention. On trials where distractors were later judged as less trustworthy, the N2pc emerged earlier, demonstrating that attention was strongly biased towards target events, and distractors were effectively inhibited. In contrast, on those trials where distractors were later judged more positively, the $\mathrm{N} 2 \mathrm{pc}$ was delayed, indicating unfocused attention to the target and less distractor suppression. This pattern of results shows that variations in attentional selectivity across trials can predict the subsequent emotional evaluation of previously ignored items that were presented together with task-relevant stimuli which had to be selectively attended. It therefore suggests that affective responses to such distractors are linked to their previous attentional inhibition.

In the studies described so far, distractor devaluation effects were observed as a result of the inhibition of distractors during attentional target selection. This raises the important question whether distractor devaluation is specifically associated with inhibitory processes that are activated during selective attention tasks, or whether similar effects might also be observed as a result of other types of inhibitory mechanisms. In the present study, we used behavioural and electrophysiological measures to investigate whether distractor devaluation can be produced as a consequence of response inhibition.
Initial behavioural evidence for a link between response inhibition and evaluative judgements comes from two recent studies (Fenske et al., 2005; Veling et al., 2008). In the Fenske et al. (2005) study, pairs of faces were presented to the left and right of fixation, and a response cue (a red or green colour patch) was flashed briefly over one of these two faces. Participants had to respond to the Go colour, but to refrain from responding when the Nogo colour was presented. Shortly afterwards, the same face pair was shown again (without the colour response cue), and participants chose the face that seemed more trustworthy or less trustworthy, on different trials. Relative to uncued faces, faces that had previously been superimposed with a Nogo colour cue were more likely to be chosen as less trustworthy, and less likely to be chosen as more trustworthy, thus suggesting that their previous association with response inhibition had resulted in an emotional devaluation. This pattern of results was not obtained when perceptually based choices were required (i.e. which face had lighter/darker background?), indicating that simple response biases provide an inadequate account of the finding. Biases of social emotional judgements were not observed for faces previously associated with Go cues, which further underlines the specific role of inhibitory processes for subsequent emotional responses. In the Veling et al. (2008) study, images taken from the International Affective Picture System (Lang et al., 1999) with positive, negative, or neutral valence were used as Go or Nogo stimuli. In a subsequent rating task, Nogo devaluation effects (i.e. lower ratings for previous Nogo images relative to previous Go or novel images) were again observed, but only for images with positive valence.

The aim of the present experiment was twofold. First, we wanted to confirm the hypothesis that response inhibition in a Go-Nogo task results in the subsequent emotional devaluation of Nogo stimuli by using a rating procedure analogous to those employed in previous studies that investigated distractor devaluation in attentional selection tasks (Fenske et al., 2004; Goolsby et al., 2008; Kiss et al., 2007; Raymond et al., 2003, 2005). Each experimental block had two parts: In the first part, participants were presented with a series of 12 novel Asian or Caucasian faces. They made manual (Go) responses to faces of one race (varied on successive blocks) and withheld responses to faces of the other race (Nogo trials). In the second part, the same 12 faces were judged successively for trustworthiness, using a 4-point scale. A set of 12 new faces was shown in every block. In contrast to Fenske et al. (2005), each face was presented individually at fixation, to eliminate any spatial attention shifts towards cued faces on the left or right side. Unlike Veling et al. (2008), who presented the same non-face images repeatedly as Go or Nogo stimuli, each individual face in our study was delivered only twice; firstly as Go or Nogo stimulus and secondly for evaluation. A single use of each stimulus in the Go-Nogo task eliminates any contamination of subsequent evaluation by mere exposure effects (Zajonc, 2001).

If response inhibition associated with Nogo faces resulted in their subsequent emotional devaluation, as suggested by the results of Fenske et al. (2005), faces that were previously presented as Nogo stimuli should be judged as less trustworthy than faces that had previously been associated with a manual response. Because the interval between the presentation of each individual face in the Go-Nogo and evaluation tasks was $\sim 30 \mathrm{~s}$, such a devaluation effect for Nogo stimuli would demonstrate that any impact of response inhibition on emotional responses can persist for an extended period of time. 
The second and main aim of the present study was to obtain electrophysiological evidence for a direct link between response inhibition and emotional devaluation by demonstrating systematic covariations between these two processes. The analysis logic applied here was analogous to the logic of our previous experiment (Kiss et al., 2007) where the N2pc component was measured as an index of attentional selectivity, except that we now employed an ERP correlate of response inhibition in GoNogo tasks. Numerous previous ERP studies have demonstrated that Nogo stimuli trigger an enhanced anterior N2 component (often termed 'Nogo N2') that is maximal at frontal recording sites (e.g. Nieuwenhuis et al., 2004; Pfefferbaum et al., 1985). This component is measured in the N2 time range (between 250 and $350 \mathrm{~ms}$ after stimulus onset) as the difference of frontal ERP N2 amplitudes between Nogo and Go stimuli.

The Nogo N2 belongs to the family of anterior N2 components that are generally assumed to reflect processes involved in top-down cognitive control (see Folstein and Van Petten, 2008, for a recent review). They originate in medial prefrontal areas, such as the anterior cingulate cortex, that are involved in topdown cognitive and motor control (e.g. Kok, 1986; Nieuwenhuis et al., 2003, 2004; Taylor et al., 2007). Several lines of evidence suggest that the Nogo N2 is specifically related to the inhibition of planned or pre-activated overt responses. Larger Nogo N2 components are triggered by non-target stimuli in overt response tasks than in tasks that involve silent counting (Pfefferbaum et al., 1985; see also Bruin and Wijers, 2002). The amplitude of this component is linked to the success of response inhibition, as it is larger for participants with low as compared to high False Alarm rates. Larger Nogo N2s are found when Go and Nogo stimuli are physically similar, in line with the view that more response inhibition is needed when non-target stimuli frequently trigger incorrect response activation (Azizian et al., 2006). It should be noted, however, that other authors (e.g. Nieuwenhuis et al., 2003; Yeung et al., 2004) have suggested that the Nogo N2 is not exclusively associated with response inhibition, but also, or even primarily, with the monitoring of conflict that is triggered by the competition between tendencies to execute or inhibit a specific response. In support of their interpretation, Nieuwenhuis et al. (2003) found that when Go stimuli were rare, and Nogo stimuli frequent, an enhanced anterior N2 was triggered by Go stimuli, suggesting that low-probability events may elicit response conflict irrespective of their status as Go or Nogo stimuli.

In the present study, we used Nogo N2 amplitudes as an index for the efficiency of response inhibition triggered by Nogo faces, based on the comprehensive evidence discussed above that this component is linked to the inhibition of motor responses. In order to minimize or eliminate the possible impact of conflict monitoring associated with the presentation of low-probability events (e.g. Nieuwenhuis et al., 2003), Go and Nogo stimuli were always equiprobable. Here, the discrimination between Go and Nogo stimuli was based on the perceptually demanding process of identifying the race of individual novel faces, which makes it likely that the speed and efficiency of response inhibition might vary considerably across trials. If the efficiency of response inhibition elicited by Nogo stimuli was directly linked to their subsequent affective devaluation, then trustworthiness ratings for Nogo stimuli should be lower on those trials where response inhibition was strong and a large Nogo N2 was elicited. In contrast, more positive trustworthiness ratings should be observed for trials with less efficient response inhibition, as reflected by a smaller Nogo N2 component.
In theory, these predictions should be tested by measuring the Nogo N2 directly on single trials, and then correlating its amplitude with the subsequent trustworthiness rating of each face. In reality, computing ERP components such as the Nogo N2 requires averaging a large number of EEG waveforms obtained on Go and Nogo trials. To accommodate this requirement, we used the trustworthiness ratings obtained for each individual face in the second evaluation part of each block as a criterion for sorting EEG responses to each face in the preceding Go-Nogo task phase (again analogous to the logic previously employed in Kiss et al., 2007). Separate ERPs in response to Go and Nogo faces were computed as a function of whether each face was subsequently rated as high ( 3 or 4 ) or low ( 1 or 2 ) in trustworthiness. When computing the Nogo N2 (a contrast between ERPs triggered in response to Go versus Nogo stimuli), it is important that Go and Nogo stimuli do not differ on any other perceptual and cognitive dimension apart from their response assignment. Therefore, we contrasted Go and Nogo ERPs separately for high-rated and low-rated stimuli. Specifically, ERPs obtained on trials with subsequently high-rated Nogo stimuli were compared to ERPs obtained on trials with subsequently high-rated Go stimuli; and the analogous comparison was conducted for low-rated stimuli ${ }^{1}$. If the efficiency of response inhibition triggered by individual Nogo faces was directly linked to their subsequent emotional devaluation, the Nogo N2 should be larger on those trials where faces were later rated as less trustworthy as compared to trials with faces that would later receive a more positive rating.

\section{MATERIALS AND METHODS \\ PARTICIPANTS}

Fifteen volunteers were paid to participate in this experiment. One of them was excluded from analyses due to EEG drift artefacts at frontal electrodes. Thus 14 participants (five male and nine female, aged between 19 and 30 years, mean age 25.7 years) remained in the sample. One participant was left-handed, the other 13 were right-handed, and all were Caucasian and had normal or corrected-to-normal vision. The experiment was approved by the Psychology Ethics Committee, Birkbeck College, and informed consent was obtained from all participants.

\section{STIMULI AND PROCEDURE}

A total of 384 colour face photographs (headshots, frontal view, neutral expression) of young adults (aged 18-39) was collected from five different databases (PAL Database, Minear and Park, 2004; AR Face Database, Martinez and Benavente, 1998; Asian Face Image Database PF01, 2001; Caltech Frontal Face Dataset, 1999; Georgia Tech Face Database, 1999). All photographs were

\footnotetext{
${ }^{1} \mathrm{~A}$ direct comparison of frontal N2 amplitudes in response to positively rated Nogo faces versus negatively rated Nogo faces is problematic because there will inevitably be low-level physical and configural differences between individual face tokens that are systematically linked with differences in their perceived trustworthiness, irrespective of their status as Go or Nogo stimuli. For example, it is conceivable that face images with a higher contrast between foreground and background, or with higher spatial frequency, are generally more (or less) likely to be judged as trustworthy. In this case, any comparison of high-rated Nogo faces versus low-rated Nogo faces would confound possible ERP correlates of response inhibition with ERP differences that are the result of those physical differences. Another related problem of directly comparing high-rated and low-rated Nogo stimuli is the possible presence of ERPs components that are directly sensitive to the affective value of individual faces.
} 
adjusted to match in size and background. Half of the faces were Asian, and the other half were Caucasian, and both sets contained an equal number of female and male faces. Stimuli were presented on a CRT monitor with $100 \mathrm{~Hz}$ refresh rate at a viewing distance of $65 \mathrm{~cm}$. Each face subtended $4.8^{\circ} \times 6.6^{\circ}$ visual angle and was presented on a light grey background $\left(18 \mathrm{~cd} / \mathrm{m}^{2}\right)$ at the centre of the screen. A black fixation cross $\left(0.4^{\circ} \times 0.4^{\circ}\right)$ was continuously present at the screen centre between face presentations. Stimulus presentation and behavioural response collection were controlled by E-Prime software (Psychology Software Tools, Pittsburgh, PA, USA).

In each block, participants performed two successive tasks (Go-Nogo and evaluation), with 12 Go-Nogo trials followed by 12 evaluation trials (see Figure 1). In the Go-Nogo task, participants were required to press the space bar with both index fingers as fast as possible whenever the displayed face was Asian (or Caucasian, in different blocks) and refrain from responding otherwise. Each face was presented for $300 \mathrm{~ms}$, followed by a $1200-m s$ empty interval until the next face appeared. In the subsequent evaluation task, participants had to judge the trustworthiness of the same 12 faces on a 4-point scale from 1 ('not at all trustworthy') to 4 ('very trustworthy') by pressing the corresponding numeric key on a standard keyboard with the index or middle finger of the left or right hand. Each face was presented for $300 \mathrm{~ms}$, followed by a 2700 -ms blank interval. The order in which individual faces were presented for evaluation was identical to that of the preceding Go-Nogo task. Prior to each run of 12 trials for the Go-Nogo and evaluation tasks, a brief instruction screen appeared, reminding participants of the task to be performed. After the final trial of the Go-Nogo task, a feedback screen informed participants about their average response time (RT) and accuracy for this block. Participants then started the next run of 12 evaluation trials by pressing a key. To ensure that the interval between seeing a face for the first time in the Go-Nogo task and seeing it again for rating was minimal, they were encouraged to start the evaluation task immediately.

For each block, individual faces were randomly drawn with the constraint that each set of 12 faces contained four Asian female, four Asian male, four Caucasian female and four Caucasian male faces. Each individual face was only shown once in each task (i.e. it was never repeated across blocks). Seven participants performed 16 blocks with Asian Go faces, followed by 16 blocks with Caucasian Go faces. This order of response assignments was reversed for the other seven participants.

\section{EEG DATA RECORDING AND ANALYSIS}

The EEG was recorded with a bandpass of $0-40 \mathrm{~Hz}$ and digitised with a sampling rate of $500 \mathrm{~Hz}$. EEG signals were recorded from 23 electrodes mounted in an elastic cap at standard scalp sites Fpz, F7, F3, Fz, F4, F8, FC5, FC6, T7, C3, Cz, C4, T8, CP5, CP6, P7, P3, Pz, P4, P8, PO7, PO8, and Oz. Horizontal eye movements (HEOG) were measured from two electrodes placed at the outer canthi of the eyes. The right earlobe was recorded as an additional channel. Impedances were kept below $5 \mathrm{k} \Omega$. Scalp electrodes were referenced to the left earlobe and re-referenced offline to averaged earlobes. Artefact rejection was performed offline by removing trials with activity exceeding $\pm 30 \mu \mathrm{V}$ in the HEOG channel (indicating leftward or rightward eye movements), $\pm 60 \mu \mathrm{V}$ at Fpz (eye blinks), and $\pm 80 \mu \mathrm{V}$ at all other electrodes (artefacts induced by movement or muscle activity). Trials with response errors (i.e. failures to respond on Go trials, or False Alarms on Nogo trials) were excluded prior to all analyses of EEG and behavioural data.

EEG waveforms obtained in response to face photographs in the Go-Nogo task were averaged relative to a $100 \mathrm{~ms}$ pre-stimulus

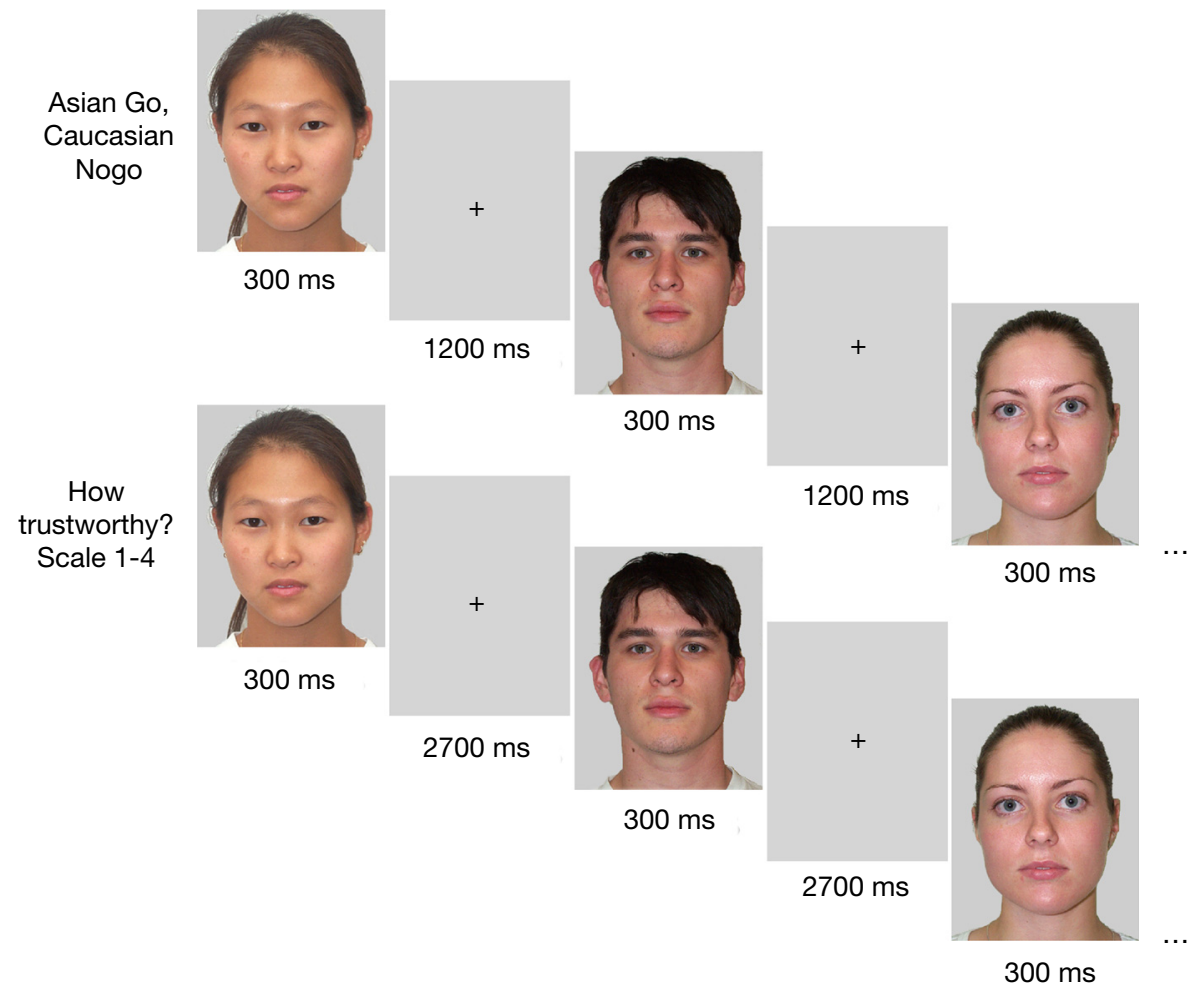

Figure 1 | Example of trial sequence for the Go-Nogo task (top panel) and the evaluation task (bottom panel). 
baseline. Before averaging, Go and Nogo trials were classified with respect to the trustworthiness rating that the specific face shown on this trial had received in the subsequent evaluation task (high: rating of 3 or 4 ; low: rating of 1 or 2$)^{2}$. Separate averages were computed for each combination of response assignment (Go versus Nogo face, collapsed across blocks with Asian Go and Caucasian Nogo faces, and vice versa), and rating (high versus low). The N2 component was quantified by computing ERP mean amplitudes within a $250-350 \mathrm{~ms}$ post-stimulus latency window. In order to compare the Nogo N2 obtained for subsequently high-rated faces with the Nogo N2 in response to subsequently low-rated faces, these mean amplitude values were then analysed in repeated-measures analyses of variance (ANOVAs) that were conducted for frontal (F3, Fz, F4) and frontopolar electrodes (F7, Fpz, F8), using the factors area (frontal versus frontopolar), response assignment, rating, and electrode site. Greenhouse-Geisser epsilon correction for deviation from sphericity was applied where appropriate.

\section{RESULTS}

\section{BEHAVIOURAL RESULTS}

The overall mean RT in correct Go trials was $489 \mathrm{~ms}(\mathrm{SD}=57)$. False Alarms occurred on 3.3\% of all Nogo trials, and participants missed $0.9 \%$ of all responses on Go trials. Mean trustworthiness ratings were calculated for each participant, for each combination of response assignment (rated face previously shown as Go versus Nogo stimulus), face race, and face gender, and were then entered into a repeated-measures ANOVA. Crucially, there was a main effect of response assignment, $F(1,13)=9.2, p<0.01$, showing that faces that had previously been presented as Nogo stimuli were rated as less trustworthy than faces that had been shown as Go stimuli (2.54 versus 2.61; see Figure 2). Response assignment

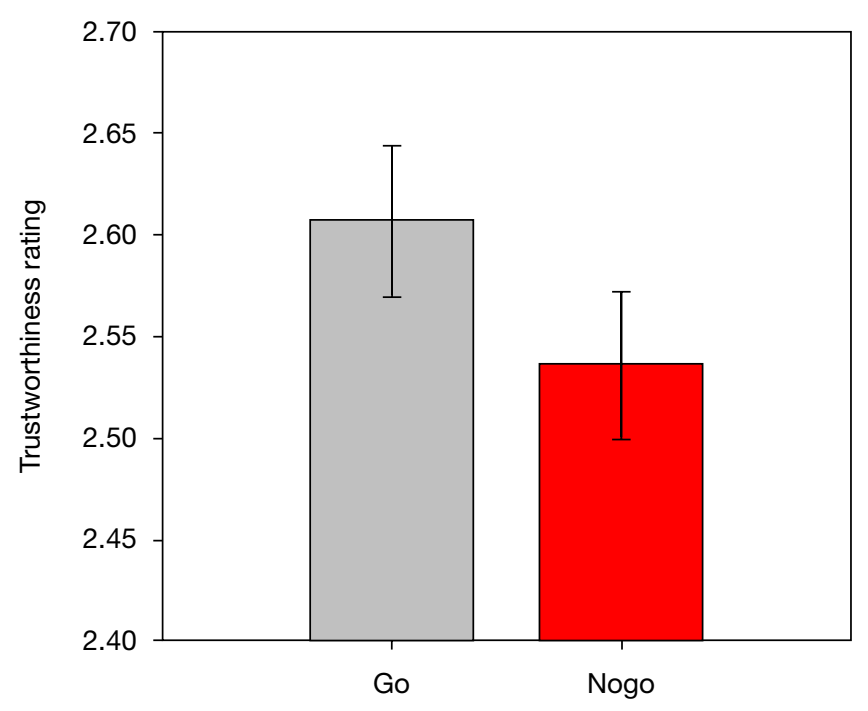

Trial type

Figure 2 | Trustworthiness ratings obtained for faces previously presented in Go and Nogo trials. Error bars represent standard errors of the mean.

${ }^{2}$ Mean ratings were 3.41 and 3.37 for the high-rated Go and Nogo faces; and 1.63 for both low-rated Go and Nogo faces. These ratings for Go faces versus Nogo faces did not differ significantly within either rating level. did not interact significantly with either race or gender (both $F<2.4$, both $p>0.145)^{3}$.

\section{ERP RESULTS - NOGO N2}

Figure 3 shows ERPs obtained at anterior, central and posterior electrodes in response to Go and Nogo faces (collapsed across Asian and Caucasian faces), separately for faces that were later rated as more trustworthy (ratings 3 and 4, right panel) or less trustworthy (ratings 1 and 2, left panel). The Nogo-Go difference waves obtained at anterior, central, and posterior electrodes for low-rated and high-rated faces are shown in Figure 4 (left panel). Figure 4 also shows scalp distribution maps of the Nogo-Go difference in the N2 time window (250-350 ms poststimulus) as a function of subsequent trustworthiness ratings (right top panels), as well as the topography of the difference of Nogo N2 amplitudes between low-rated and high-rated faces (right bottom panel). These figures show an enhanced anterior N2 in response to Nogo faces as compared to Go faces, as expected. Critically, the amplitude of this Nogo N2 appears larger in response to faces that had later received a low trustworthiness rating than for faces that were rated more positively. This modulation of the Nogo N2 as a function of subsequent ratings shows a distinct frontal topography (Figure 4, right panel).

These observations were substantiated by statistical analyses. The initial omnibus ANOVA performed on mean amplitudes in the $\mathrm{N} 2$ time window (250-350 ms post-stimulus) showed a main effect of response assignment, $F(1,13)=25.8, p<0.001$, indicating that the anterior $\mathrm{N} 2$ for Nogo faces was enhanced relative to Go faces (see Figure 3). A response assignment $\times$ rating $\times$ area interaction was also found, $F(1,13)=9.8, p<0.01$. To explore this interaction, separate ANOVAs were conducted for frontal and frontopolar electrodes. A main effect of response assignment was observed for frontal electrodes, $F(1,13)=22.9, p<0.001$, confirming the presence of a Nogo N2. Most importantly, there was a significant interaction between response assignment and rating at frontal electrodes, $F(1,13)=4.7, p<0.05$, demonstrating that the Nogo N2 component (i.e. the difference between ERPs on Nogo as compared to Go trials) was significantly larger for faces that were subsequently rated as less trustworthy as compared to high-rated faces. Although the difference between Nogo N2 amplitudes elicited by high-rated faces versus lowrated faces was numerically larger over the right hemisphere (see Figure 4, right panel), a separate ANOVA performed only on lateral frontal electrodes F3/4 yielded no significant response assignment $\times$ rating $\times$ hemisphere interaction, $F(1,13)=2.3$, $p=0.15$. At frontopolar electrodes, a main effect of response assignment, $F(1,13)=27.3, p<0.001$, reflecting the presence of the Nogo N2 component, was accompanied by a significant response assignment $\times$ electrode site interaction, $F(2,26)=15.7$, $p<0.001, \varepsilon=0.708$, as the Nogo-Go difference was largest at Fpz and smallest at F7 (see Figure 4). Frontopolar Nogo N2 amplitudes were also numerically larger for low-rated relative to high-rated faces, but in contrast to the results observed at frontal

${ }^{3}$ In addition, a main effect of race was obtained, $F(1,13)=6.9, p<0.05$, as Asian faces were generally rated as more trustworthy than Caucasian faces (mean ratings of 2.67 and 2.48, respectively). A main effect of gender, $F(1,13)=60.7$, $p<0.001$, showed that female faces were generally rated as more trustworthy than male faces (mean ratings: 2.87 versus 2.28). A race $\times$ gender interaction, $F(1,13)=40.4, p<0.001$, indicated that this bias in favour of females was more pronounced for Asian faces (3.09 versus 2.25) than for Caucasian faces (2.65 versus 2.30$)$. 


\section{Low rating}
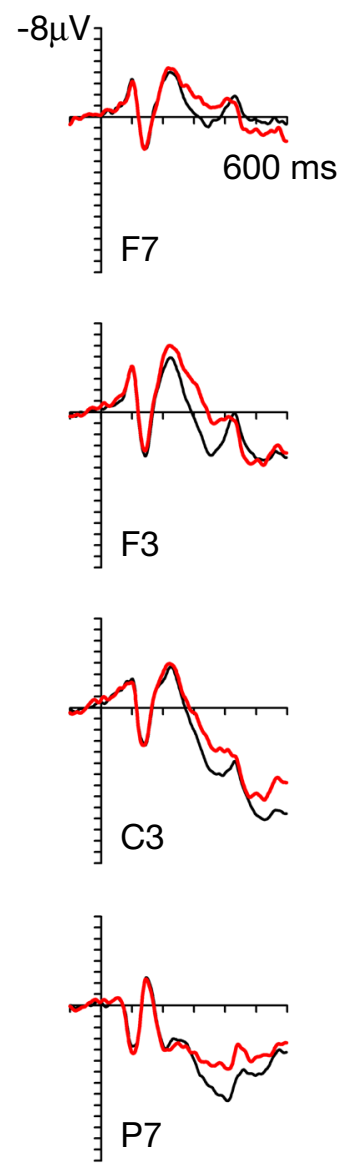
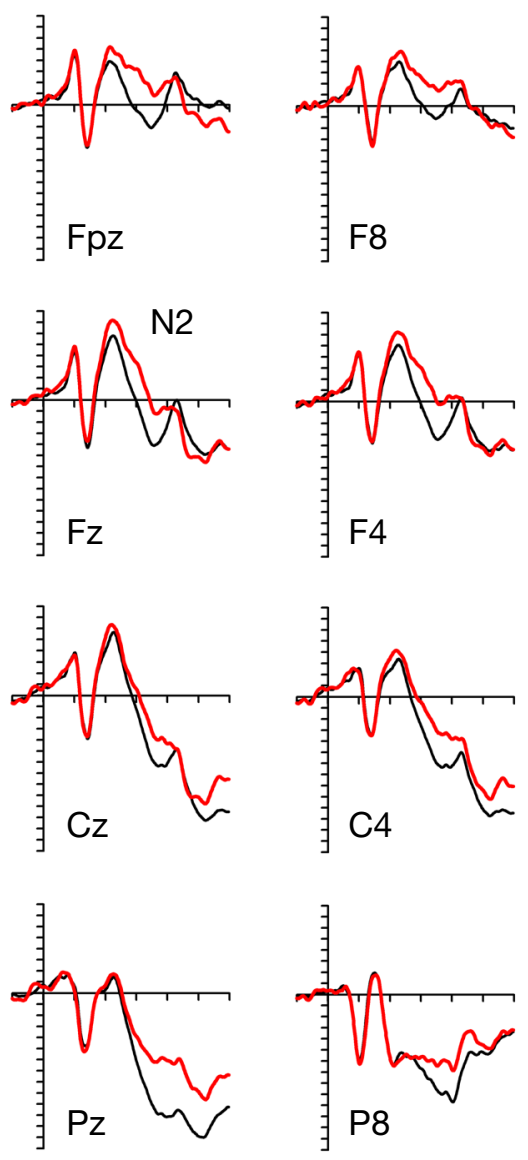
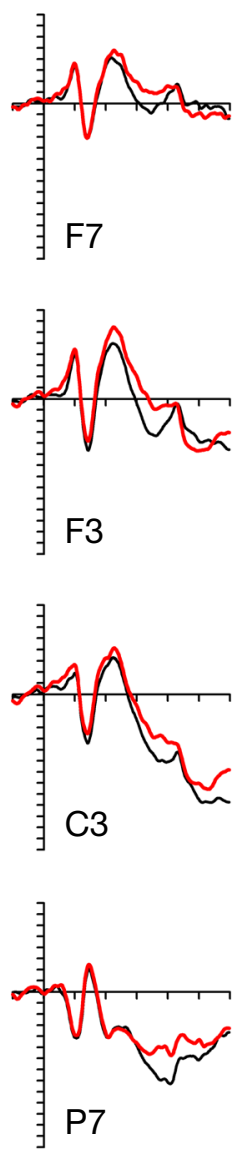

High rating
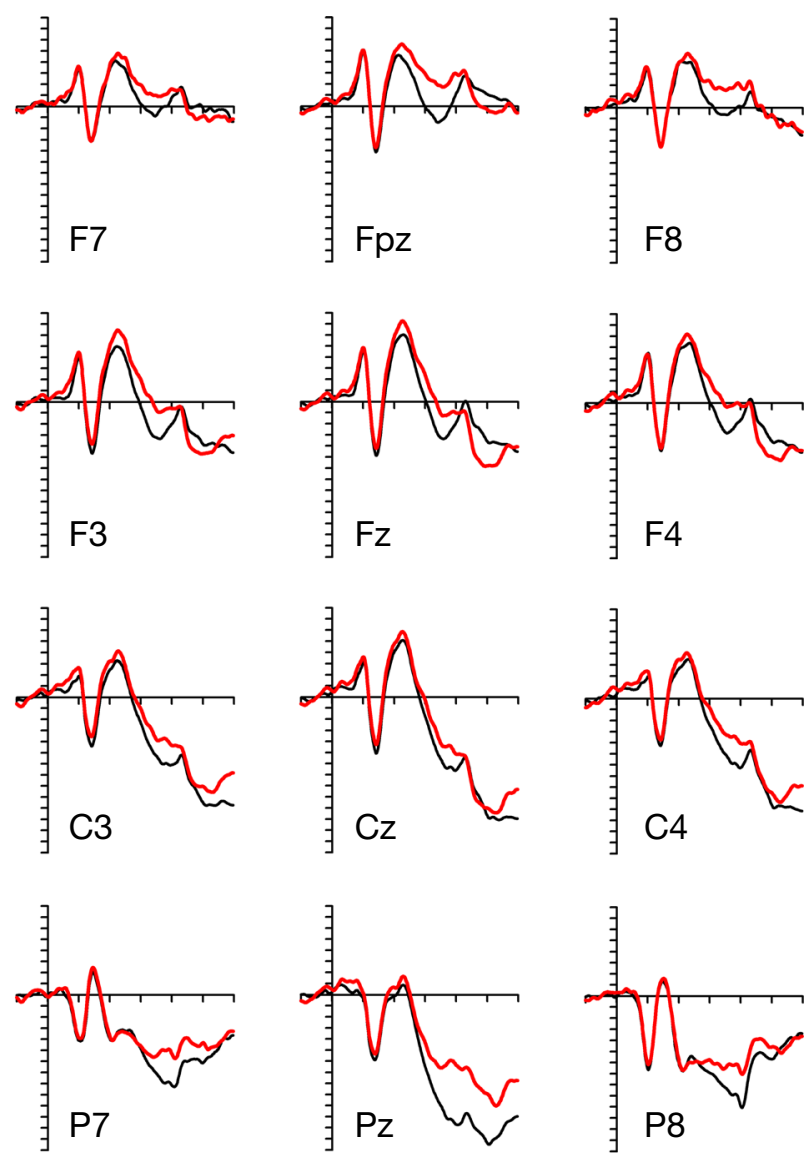

Go

Nogo

Figure 3 | Grand-averaged ERPs elicited at frontopolar, frontal, central, and parietal electrode sites in Go trials (black line) and Nogo trials (red line) displayed separately for faces that subsequently received a low trustworthiness rating (left panels) and a high trustworthiness rating (right panels). For display purposes only, waveforms were low-pass filtered at $40 \mathrm{~Hz}$ to remove any residual high-frequency noise.

electrodes, there was no significant interaction between response assignment and rating, $F(1,13)=1.0, p=0.331$.

\section{DISCUSSION}

The current experiment provides converging behavioural and electrophysiological evidence for the hypothesis that response inhibition triggered by Nogo stimuli has a systematic impact on their subsequent emotional evaluation. Faces that had previously been presented as Nogo stimuli were later rated significantly lower in terms of their trustworthiness than previous Go faces. This devaluation occurred in spite of the fact that the time interval separating the presentation of individual faces in the Go-Nogo and evaluation task was relatively long (about $30 \mathrm{~s}$ ), and included 12 different face images that required response selection or evaluation. This suggests that effects of response inhibition on affective evaluation are robust and persist over an extended period of time. Overall, the current behavioural results confirm and extend previous findings by Fenske et al. (2005) and Veling et al. (2008), who also observed behavioural devaluation effects as a consequence of response inhibition.
Having demonstrated a reliable difference in trustworthiness ratings as a function of whether a face had previously been encountered as a Go or Nogo stimulus, the main objective of the present study was to obtain independent ERP evidence for a direct link between response inhibition and subsequent emotional devaluation. As expected, a frontally maximal negative component was triggered by Nogo faces relative to Go faces in the N2 time range (see Figures 3 and 4), in line with numerous previous studies that have found this Nogo N2 in the context of Go-Nogo tasks (e.g. Eimer, 1993; Jodo and Kayama, 1992; Kok, 1986; Nieuwenhuis et al., 2003, 2004; Pfefferbaum et al., 1985). As the currently available evidence supports the view that the Nogo $\mathrm{N} 2$ is associated with top-down response inhibition [see Section 'Introduction', but see also Nieuwenhuis et al. (2003) and Yeung et al. (2004), for an alternative view], the hypothesis that response inhibition is directly linked to the subsequent devaluation of Nogo stimuli would predict an association between the strength of response inhibition, as reflected by the amplitude of the Nogo N2, and the strength of the devaluation effect, as manifested by subsequent trustworthiness ratings. To test this prediction, we computed separate ERPs for Go and Nogo faces 


\section{Nogo-Go difference}
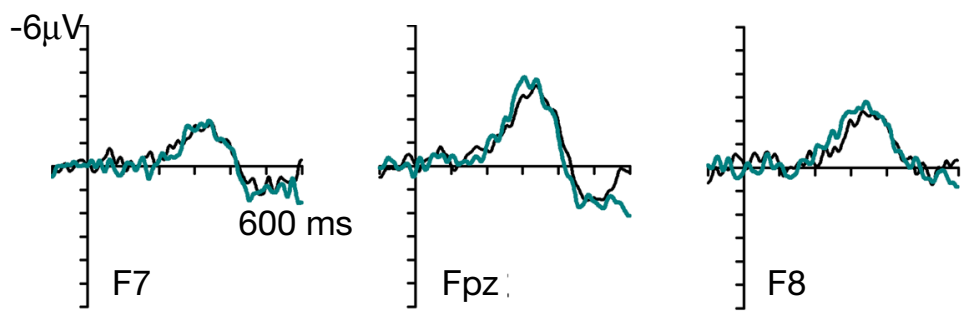

Nogo-Go
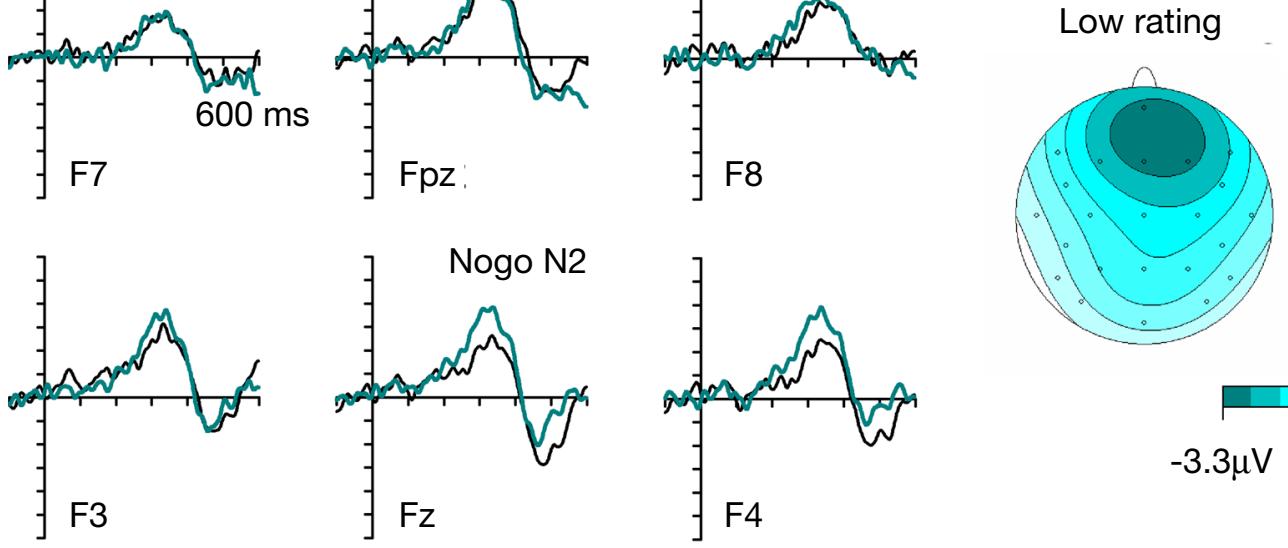

High rating
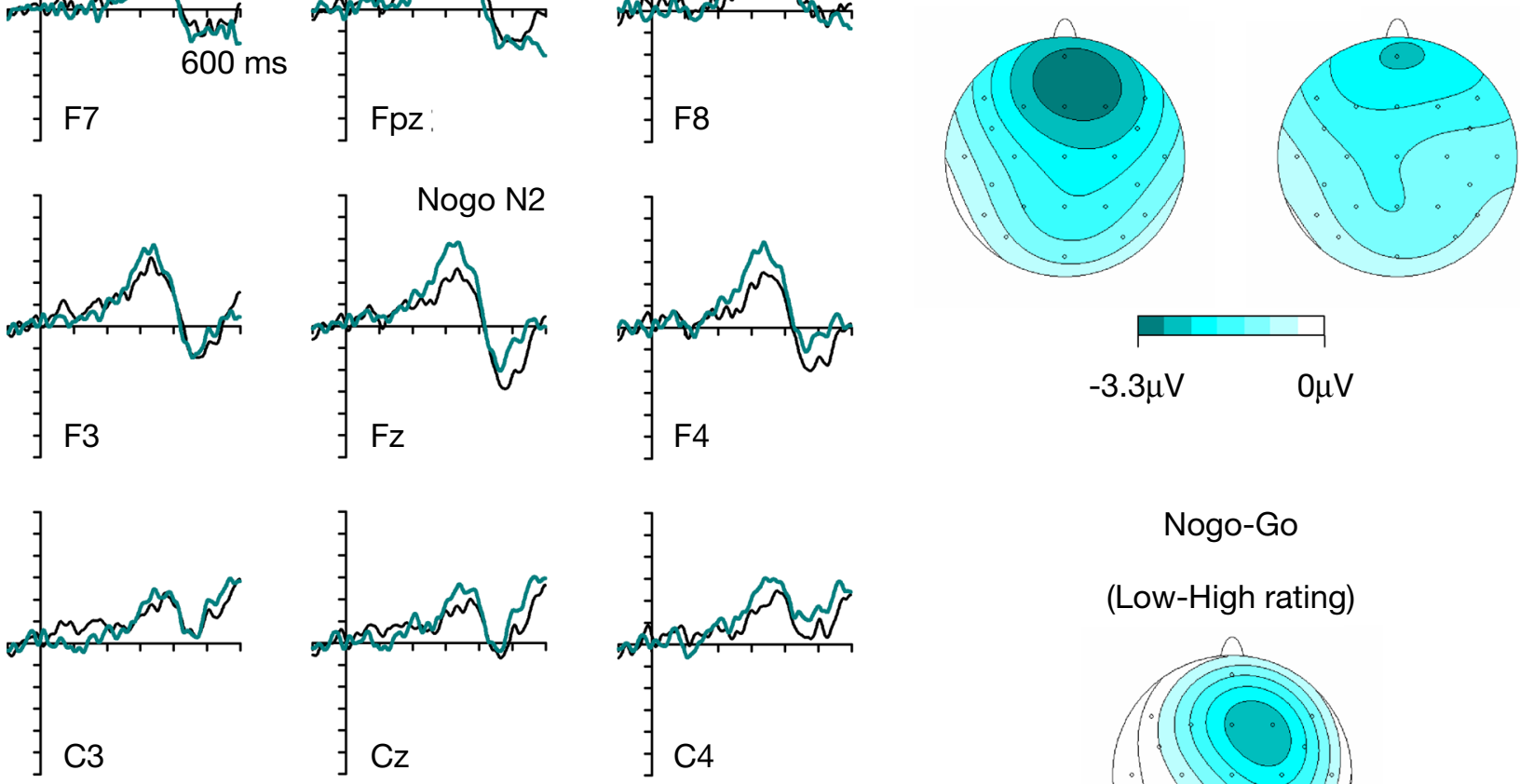

Nogo-Go
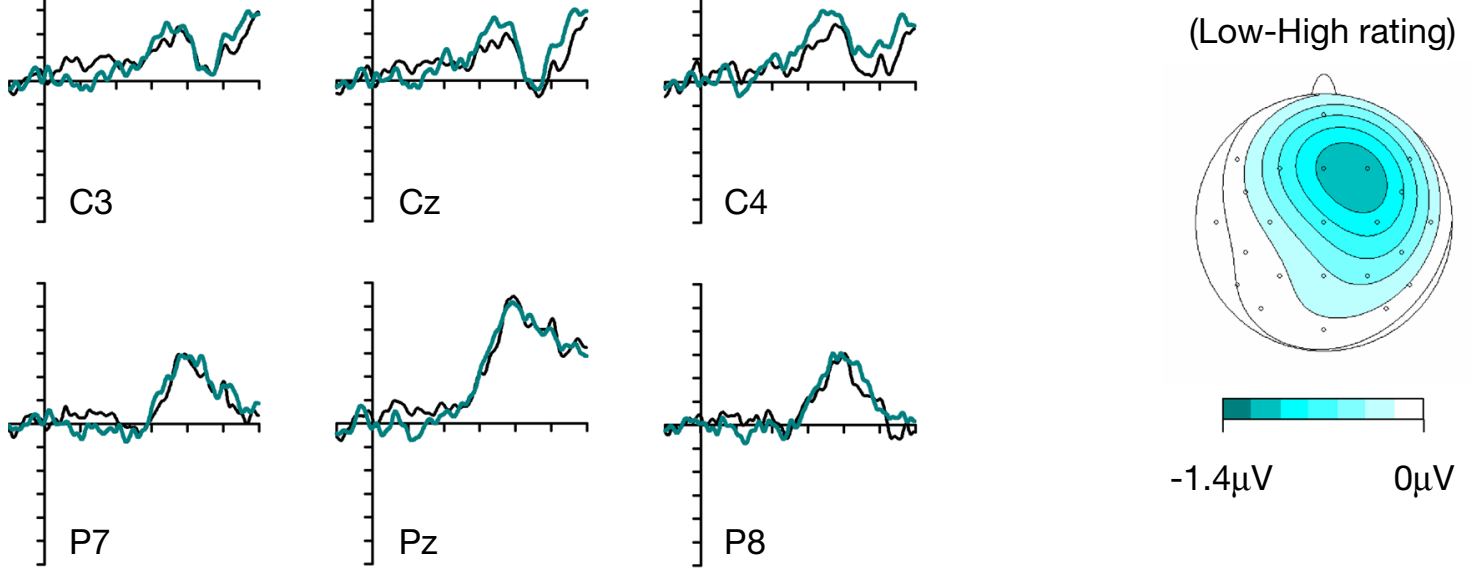

\section{High rating \\ Low rating}

Figure 4 | Left panels show difference waveforms obtained by subtracting Go trials from Nogo trials, separately for faces that subsequently received a high (black line) or low (dark cyan line) trustworthiness rating. Right panels show topographical maps of Nogo-Go difference waveforms obtained in the Nogo N2 time window (250-350 ms post-stimulus) for faces that were later judged as low or high in their trustworthiness. Bottom right panel: Scalp topography of the difference of the Nogo N2 in the same time window as a function of subsequent trustworthiness ratings, computed by subtracting Nogo-Go difference waveforms for high-rated faces from Nogo-Go difference waveforms for low-rated faces.

as a function of whether these faces were later judged as high or low in terms of their trustworthiness. If stronger response activation leads to more pronounced affective devaluation, the Nogo N2 should be larger for faces that are later rated as less trustworthy than for faces that are subsequently judged more positively. This was exactly the pattern of results obtained in this experiment: Although the Nogo N2 was clearly present both for low-rated and high-rated faces, it was significantly larger for faces that were later judged as less trustworthy. Importantly, the anterior topography of this differential effect was similar to the scalp distribution of the Nogo N2 proper (see Figure 4, right panel). This further supports the hypothesis that differences in Nogo N2 amplitudes that are associated with subsequent affective judgements reflect a difference in the strength of cortical response inhibition processes that are mediated by medial prefrontal areas involved in top-down motor control.

Distractor devaluation effects previously found as a result of the selection of targets among distractors in visual search tasks (Fenske et al., 2004; Raymond et al., 2003, 2005) have been interpreted as evidence that the attentional inhibition of irrelevant distractors is encoded along with the representation of other stimulus features, and that this inhibitory tag will affect subsequent affective judgements. The present results, together with earlier findings by Fenske et al. (2005) and Veling et al. (2008), demonstrate that such devaluation processes do not only operate in the context of attentional selection tasks, but are also activated as a 
consequence of cortical response inhibition. A parsimonious way to interpret these new findings from Go-Nogo experiments is to assume that inhibitory tags of the kind postulated by Raymond and colleagues are generated and encoded in a wide variety of task contexts that involve top-down inhibition of visual objects, and will therefore produce similar effects on affective evaluation regardless of whether inhibition is located at perceptual, higher cognitive, or response-related stages of processing.

Perhaps cortical inhibition of any type that has been directed at the representation of a visual object is used to reduce the predicted value code associated with that object. An emerging picture of value coding mechanisms in the brain indicates that a complex circuit involving prefrontal cortical areas, especially the orbitofrontal cortex; the ventral striatum, including the nucleus accumbens (O'Doherty et al., 2002); and the amygdala (Gottfried et al., 2003) is used to flexibly provide the brain with a mechanism to predict the value of responding (in a particular way) to specific stimuli, or classes of stimuli. Value prediction mechanisms, especially the amygdala (Paton et al., 2006), appear to be highly updatable, using cumulative prediction errors to enable value learning. If learned value prediction codes are available to the affective mechanisms that control explicit rating behaviour and these codes can be modulated by cortical inhibition, then the value prediction system could be the neural mechanism linking inhibition and affective evaluation.

It should be noted that in their previous investigation of the effects of response inhibition on the evaluation of non-face images, Veling et al. (2008) found devaluation effects only for affectively positive Nogo stimuli, but not for affectively neutral or negative images. Based on these results, these authors proposed a more domain-specific motor account of affective devaluation. They suggested that the function of affective devaluation is to counteract behavioural approach tendencies towards positively valenced stimuli under conditions where such tendencies could result in response errors, thereby minimizing response conflict. Future studies will need to contrast and compare distractor devaluation effects in different task contexts in order to address the question whether such effects are a general, domainunspecific result of top-down inhibition, or whether there are important functional differences between such effects in perceptual-attentional, cognitive, and motor tasks.

In summary, the central new finding of the present experiment is the discovery of a systematic covariation between independent electrophysiological markers of response inhibition in a Go-Nogo task, and behavioural measures of affective evaluation. The efficiency of response inhibition triggered by visual stimuli, as reflected by the amplitude of the anterior Nogo N2 component, was found to be closely associated with subsequent affective responses to these stimuli. This demonstrates that topdown inhibition in motor control has immediate consequences for the affective evaluation of visual stimuli, and suggests close links between response inhibition and emotion.

\section{CONFLICT OF INTEREST STATEMENT}

The authors declare that the research was conducted in the absence of any commercial or financial relationships that could be construed as a potential conflict of interest.

\section{ACKNOWLEDGEMENTS}

This study was supported by a grant from the Biotechnology and Biological Sciences Research Council (BBSRC), UK. Martin
Eimer holds a Royal Society-Wolfson Research Merit Award. Our thanks to Tamara Cristescu for technical assistance.

\section{REFERENCES}

Asian Face Image Database PF01 (2001). Collected at the Intelligent Multimedia Lab, Pohang University of Science and Technology, Korea. Available online at: http://nova.postech.ac.kr/ dkim/new_imlab/

Azizian, A., Freitas, A. L., Parvaz, M. A., and Squires, N. K. (2006). Beware misleading cues: perceptual similarity modulates the N2/P3 complex. Psychophysiology 43, 253-260.

Bruin, K. J., and Wijers, A. A. (2002). Inhibition, response mode, and stimulus probability: a comparative event-related potential study. Clin. Neurophysiol. $113,1172-1182$.

Bush, G., Luu, P., and Posner, M. I. (2000). Cognitive and emotional influences in anterior cingulate cortex. Trends Cogn. Sci. 4, 215-222.

Cacioppo, J. T., and Gardner, W. L. (1999). Emotion. Annu. Rev. Psychol. 50, 191-214.

Caltech Frontal Face Dataset (1999). Collected by Markus Weber at California Institute of Technology, USA. Available online at: http://www.vision.caltech. edu/html-files/archive.html

Chen, Y, Martinez-Conde, S., Macknik, S. L., Bereshpolova, Y., Swadlow, H. A. and Alonso, J.-M. (2008). Task difficulty modulates the activity of specific neuronal populations in primary visual cortex. Nat Neurosci. 11, 974-982.

Eastwood, J. D., Smilek, D., and Merikle, P. M. (2001). Differential attentional guidance by unattended faces expressing positive and negative emotion. Percept. Psychophys. 63, 1004-1013.

Eimer, M. (1993). ERPs elicited by Go and Nogo stimuli: effects of attention and stimulus probability. Biol. Psychol. 35, 123-138.

Eimer, M. (1996). The N2pc component as an indicator of attentional selectivity. Electroencephalogr. Clin. Neurophysiol. 99, 225-234.

Esteves, F., Parra, C., Dimberg, U., and Öhman, A. (1994). Nonconscious associative learning: Pavlovian conditioning of skin conductance responses to masked fear-relevant facial stimuli. Psychophysiology 31, 375-385.

Fenske, M. J., Raymond, J. E., Kessler, K., Westoby, N., and Tipper, S. P. (2005). Attentional inhibition has social-emotional consequences for unfamiliar faces. Psychol. Sci. 16, 753-758.

Fenske, M. J., Raymond, J. E., and Kunar, M. A. (2004). The affective consequences of visual attention in preview search. Psychon. Bull. Rev. 11, 1055-1061.

Folstein, J. R., and Van Petten, C. (2008). Influence of cognitive control and mismatch on the N2 component of the ERP: a review. Psychophysiology 45 $152-170$.

Fox, E., Russo, R., Bowles, R., and Dutton, K. (2001). Do threatening stimuli draw or hold visual attention in subclinical anxiety? J. Exp. Psychol. Gen. 130, 681-700.

Georgia Tech Face Database (1999). Collected at the Georgia Institute of Technology, USA. Available online at: http://www.anefian.com/face_reco.htm

Goolsby, B. A., Raymond, J. E., Silvert, L., Kiss, M., Fragopanagos, N., Taylor, J. G., Eimer, M., Nobre, A. C., and Shapiro, K. L. (2008). Feature-based inhibition underlies the affective consequences of attention. Vis. Cogn., Advance online publication, doi: 10.1080/13506280801904095

Gottfried, J. A., O'Doherty, J., and Dolan, R. J. (2003). Encoding predictive reward value in human amygdala and orbitofrontal cortex. Science 301, 1104-1107.

Griffiths, O., and Mitchell, C. J. (2008). Negative priming reduces affective ratings. Cogn. Emot. 22, 1119-1129.

Jodo, E., and Kayama, Y. (1992). Relation of a negative ERP component to response inhibition in a go/no-go task. Electroencephalogr. Clin. Neurophysiol. $82,477-482$.

Kessler, K., and Tipper, S. P. (2004). Retrieval of implicit inhibitory processes: the impact of visual field, object-identity, and memory dynamics. Vis. Cogn. 11, 965-995.

Kiss, M., Goolsby, B. A., Raymond, J. E., Shapiro, K. L., Silvert, L., Nobre, A. C., Fragopanagos, N., Taylor, J. G., and Eimer, M. (2007). Efficient attentional selection predicts distractor devaluation: ERP evidence for a direct link between attention and emotion. J. Cogn. Neurosci. 19, 1316-1322.

Kok, A. (1986). Effects of degradation of visual stimuli on components of the eventrelated potential (ERP) in go/no-go reaction tasks. Biol. Psychol. 23, 21-38.

Lang, P. J., Bradley, M. M., and Cuthbert, B. N. (1990). Emotion, attention, and the startle reflex. Psychol. Rev. 97, 377-395.

Lang, P. J., Bradley, M. M., and Cuthbert, B. N. (1997). Motivated attention: affect, activation and action. In Attention and Orienting: Sensory and Motivational Processes, P. J. Lang, R. F. Simons and M. T. Balaban, eds (Hillsdale, NJ, Lawrence Erlbaum Associates, Inc.), pp. 97-135.

Lang, P. J., Bradley, M. M., and Cuthbert, B. N. (1999). International affective picture system (IAPS): instruction manual and affective ratings. The Center for Research in Psychophysiology, University of Florida.

Lang, P. J., Bradley, M. M., Fitzsimmons, J. R., Cuthbert, B. N., Scott, J. D., Moulder, B., and Nangia, V. (1998). Emotional arousal and activation of the visual cortex: an fMRI analysis. Psychophysiology 35, 199-210. 
Luck, S. J., and Hillyard, S. A. (1994). Spatial filtering during visual search: evidence from human electrophysiology. J. Exp. Psychol. Hum. Percept. Perform. 20, 1000-1014.

Martinez, A. M., and Benavente, R. (1998). The AR Face Database. CVC Technical Report 24.

Minear, M., and Park, D. C. (2004). A lifespan database of adult facial stimuli. Behav. Res. Methods Instrum. Comput. 36, 630-633.

Mogg, K., and Bradley, B. P. (1999). Orienting of attention to threatening facia expressions presented under conditions of restricted awareness. Cogn. Emot. $13,713-740$.

Nieuwenhuis, S., Yeung, N., and Cohen, J. D. (2004). Stimulus modality, perceptual overlap, and the go/no-go N2. Psychophysiology 41, 157-160.

Nieuwenhuis, S., Yeung, N., Van den Wildenberg, W., and Ridderinkhof, K. R. (2003). Electrophysiological correlates of anterior cingulate function in a go/no-go task: effects of response conflict and trial-type frequency. Cogn Affect Behav. Neurosci. 3, 17-26.

O'Doherty, J. P., Deichmann, R., Critchley, H. D., and Dolan, R. J. (2002). Neural responses during anticipation of a primary taste reward. Neuron 33, 815-826.

Öhman, A., Flykt, and Esteves, F. (2001). Emotion drives attention: detecting the snake in the grass. J. Exp. Psychol. Gen. 130, 466-478.

Paton, J. J., Belova, M. A., Morrison, S. E., and Salzman, C. D. (2006). The primate amygdala represents the positive and negative value of visual stimuli during learning. Nature 439, 865-870.

Pessoa, L., and Ungerleider, L. G. (2004). Neuroimaging studies of attention and the processing of emotion-laden stimuli. Prog. Brain Res. 144, 171-182.

Pfefferbaum, A., Ford, J. M., Weller, B. J., and Kopell, B. S. (1985). ERPs to response reduction and inhibition. Electroencephalogr. Clin. Neurophysiol. 60, 423-434.
Raymond, J. E., Fenske, M. J., and Tavassoli, N. T. (2003). Selective attention determines emotional responses to novel visual stimuli. Psychol. Sci. 14, 537-542.

Raymond, J. E., Fenske, M. J., and Westoby, N. (2005). Emotional devaluation of distracting patterns and faces: a consequence of attentional inhibition during visual search? J. Exp. Psychol. Hum. Percept. Perform. 31, 1404-1415.

Reber, R., Winkielman, P., and Schwarz, N. (1998). Effects of perceptual fluency on affective judgments. Psychol. Sci. 9, 45-48.

Taylor, P. C. J., Nobre, A. C., and Rushworth, M. F. S. (2007). Subsecond changes in top-down control exerted by human medial frontal cortex during conflict and action selection: a combined transcranial magnetic stimulationelectroencephalography study. J. Neurosci. 27, 11343-11353.

Tipper, S. P., Grison, S., and Kessler, K. (2003). Long-term inhibition of return of attention. Psychol. Sci. 14, 19-25.

Veling, H., Holland, R. W., and van Knippenberg, A. (2008). When approach motivation and behavioral inhibition collide: behavior regulation through stimulus devaluation. J. Exp. Soc. Psychol. 44, 1013-1019.

Vuilleumier, P., Armony, J. L., Driver, J., and Dolan, R. J. (2001). Effects of attention and emotion on face processing in the human brain: an event-related fMRI study. Neuron 30, 829-841.

Woodman, G. F., and Luck, S. J. (1999). Electrophysiological measurement of rapid shifts of attention during visual search. Nature 400, 867-869.

Yeung, N., Botvinick, M. M., and Cohen, J. D. (2004). The neural basis of error detection: conflict monitoring and the error-related negativity. Psychol. Rev. $111,931-959$.

Zajonc, R. B. (2001). Mere exposure: a gateway to the subliminal. Curr. Dir. Psychol. Sci. 10, 224-228. 